\title{
Chemotherapy research for metastatic prostate cancer
}

\author{
Anil Kapoor, MD, FRCSC;' Sebastien J. Hotte, MD, FRCPC ${ }^{2}$ \\ 'Professor of Surgery (Urology), McMaster University, Associate Editor (Oncology), Canadian Urological Association Journal (CUAJ), Hamilton, ON, Canada; ${ }^{2}$ Associate Professor, Department of Oncology, \\ Division of Medical Oncology, McMaster University; Medical Oncologist, Juravinski Cancer Centre, Hamilton, ON, Canada
}

Cite as: Can Urol Assoc J 2016;10(7-8-Supp|3):S140-3 htrp://dx.doi.org/10.5489/cuai.4055

Declines in quality of life with early docetaxel treatment may be offset by long-term gains for men with metastatic hormone-sensitive prostate cancer

In 2004, docetaxel was recognized as the first agent to confer an overall survival (OS) benefit in men with metastatic prostate cancer; ${ }^{1,2}$ however, docetaxel is also associated with known side effects that can diminish quality of life (QOL). ${ }^{3,4}$ The CHAARTED study, which showed an OS benefit of docetaxel in metastatic, hormone-sensitive prostate cancer, ${ }^{5}$ also included global measures of QOL that account for disease-related symptoms, as well as treatment-related symptoms. Dr. Linda Patrick-Miller presented the QOL results from CHAARTED at ASCO 2016. ${ }^{6}$ The 790 patients in CHAARTED who were randomly assigned to androgendeprivation therapy (ADT) plus docetaxel $(n=397)$ or ADT alone $(n=393)$ underwent QOL assessment at baseline, three, six, nine, and 12 months following randomization. Compared with those who received ADT alone, the patients who received the combination of ADT plus docetaxel had significantly worse overall QOL, as measured by Functional Assessment of Cancer Therapy-Prostate (FACT-P) scores, at three months $(p=0.02)$; however, by Month 12 , their FACT-P scores were significantly better $(p=0.04)$. As expected, fatigue was significantly worse at three months in the docetaxel group, as measured by Functional Assessment of Chronic IIIness Therapy-Fatigue (FACIT-F) scores $(p<0.001)$; however, by six months, FACIT-F scores had returned to baseline and were no different from those who received ADT alone. FACT-Taxane scores were significantly worse in the docetaxel group at all time points, but Brief Pain Inventory (BPI) scores did not differ between the two groups at any time. Emotional well-being was significantly greater in the docetaxel group at all time points. Coupled with the OS benefit observed in metastatic hormone-sensitive prostate cancer, these results suggest that early docetaxel treatment offers a clinically meaningful benefit in men with newly diagnosed advanced prostate cancer.

Intermittent docetaxel is non-inferior to continuous docetaxel treatment in one-year survival of men with metastatic castrate-resistant prostate cancer.

Intermittent docetaxel treatment offers patients the benefit of a "treatment holiday" — reducing their overall exposure to docetaxel and cumulative toxicity, and potentially delaying resistance to taxanes. ${ }^{7}$ However, data on the noninferiority of intermittent vs. continuous docetaxel in men with castrate-resistant prostate cancer (CRPC) are lacking. The PRINCE study randomly assigned men with chemotherapy-naïve metastatic CRPC (mCRPC) to either intermittent $(n=78)$ or continuous $(n=78)$ treatment with docetaxel. ${ }^{8}$ Men in the intermittent treatment arm were started on a 12-week sequence of either four cycles given in a three-weekly regimen or three cycles in a weekly regimen, followed by a treatment holiday until disease progression. The continuous arm received docetaxel in either a three-weekly or weekly regimen until death. Patients in the intermittent arm spent a median time of 15 weeks on a treatment holiday (range 1-69 weeks), translating to $38 \%$ of the overall treatment duration. One-year survival was similar between the intermittent and continuous treatment arms (75.8\% vs. $72.6 \%)$ and met the non-inferiority criteria. However, the difference in median OS (18.3 months vs. 19.3 months) did not meet the non-inferiority criteria, according to a post-hoc analysis. Differences in progression-free survival (PFS) and time to treatment failure were not significant between the two groups and the safety profiles of both study arms were comparable. The PRINCE study was limited by poor recruitment, resulting in a power of only $39 \%$ of the planned study. Although results of a study with such a small sample size cannot be used to determine whether intermittent docetaxel is truly non-inferior to continuous treatment, these results still suggest that intermittent docetaxel may present a treatment option for patients with mCRPC. 


\section{Cabazitaxel trials presented at ASCO 2016 show similar overall survival to docetaxel and maintenance of survival benefits with lower dose}

The phase 3 TROPIC study showed that cabazitaxel plus prednisone significantly improves OS in men with mCRPC who have previously been treated with a docetaxel-containing regimen compared with mitoxantrone plus prednisone. ${ }^{9}$ Building on these results, the multinational, open-label, phase 3 FIRSTANA study examined whether cabazitaxel plus prednisone is superior to docetaxel plus prednisone in chemotherapy-naïve mCRPC. ${ }^{10}$ The first trial to compare two life-prolonging therapies in mCRPC, FIRSTANA enrolled patients with mCRPC and Eastern Cooperative Oncology Group (ECOG) performance status of 0-2 who had progressed after castration. Patients were randomly assigned in $1: 1: 1$ ratio to cabazitaxel $20 \mathrm{mg} / \mathrm{m}^{2}$ every three weeks $(n=391)$, cabazitaxel $25 \mathrm{mg} / \mathrm{m}^{2}$ every three weeks $(n=389)$, or docetaxel $75 \mathrm{mg} / \mathrm{m}^{2}(\mathrm{n}=388)$ - all given every three weeks with prednisone $10 \mathrm{mg} /$ day.

Median OS, the primary endpoint of the study, did not differ among the three groups (24.5 months for cabazitaxel $20 \mathrm{mg} / \mathrm{m}^{2}, 25.2$ months for cabazitaxel $25 \mathrm{mg} / \mathrm{m}^{2}$, and 24.3 months for docetaxel $75 \mathrm{mg} / \mathrm{m}^{2}$ ) (Fig. 1). The secondary endpoint PFS (based on tumour, prostate-specific antigen [PSA], pain progression, or death) also did not differ significantly among the three groups (4.4 months for cabazitaxel $20 \mathrm{mg} / \mathrm{m}^{2}, 5.1 \mathrm{months}$ for cabazitaxel $25 \mathrm{mg} / \mathrm{m}^{2}$, and 5.3 months for docetaxel $75 \mathrm{mg} / \mathrm{m}^{2}$ ). The Response Evaluation Criteria In Solid Tumours (RECIST) response rate was significantly better in the cabazitaxel $25 \mathrm{mg} / \mathrm{m}^{2}$ group than in the docetaxel $75 \mathrm{mg} / \mathrm{m}^{2}$ group ( $41.6 \%$ vs. $30.9 \%$; $\left.\mathrm{p}=0.0370\right)$. Other secondary endpoints, including PSA response rate, did not significantly differ across groups.

While no new safety concerns were identified, differences in toxicity profiles among the taxanes were noted. Treatment-emergent adverse events (TEAEs) were less frequent across most categories in the cabazitaxel $20 \mathrm{mg} / \mathrm{m}^{2}$ group. Febrile neutropenia, diarrhea, and hematuria were more frequent in men treated with cabazitaxel $25 \mathrm{mg} / \mathrm{m}^{2}$, while peripheral neuropathy, peripheral edema, alopecia, and nail disorders were more frequent in men treated with docetaxel.

Determining the right dose for the right patient remains a significant challenge for many drugs used in oncology today, including cabazitaxel. Results of the open-label PROSELICA study, exploring the inferiority of a slightly lower dose of cabazitaxel, $20 \mathrm{mg} / \mathrm{m}^{2}$, were presented by de Bono and colleagues at ASCO 2016. ${ }^{11}$ This multinational, phase 3 study involved 1200 men with mCRPC and ECOG performance status $0-2$, who had progressed after treatment with docetaxel. The men were randomly assigned in a $1: 1$ ratio to either $20 \mathrm{mg} / \mathrm{m}^{2}$ or $25 \mathrm{mg} / \mathrm{m}^{2}$ of cabazitaxel plus prednisone, with the hypothesis that the $20 \mathrm{mg} / \mathrm{m}^{2}$ dose would

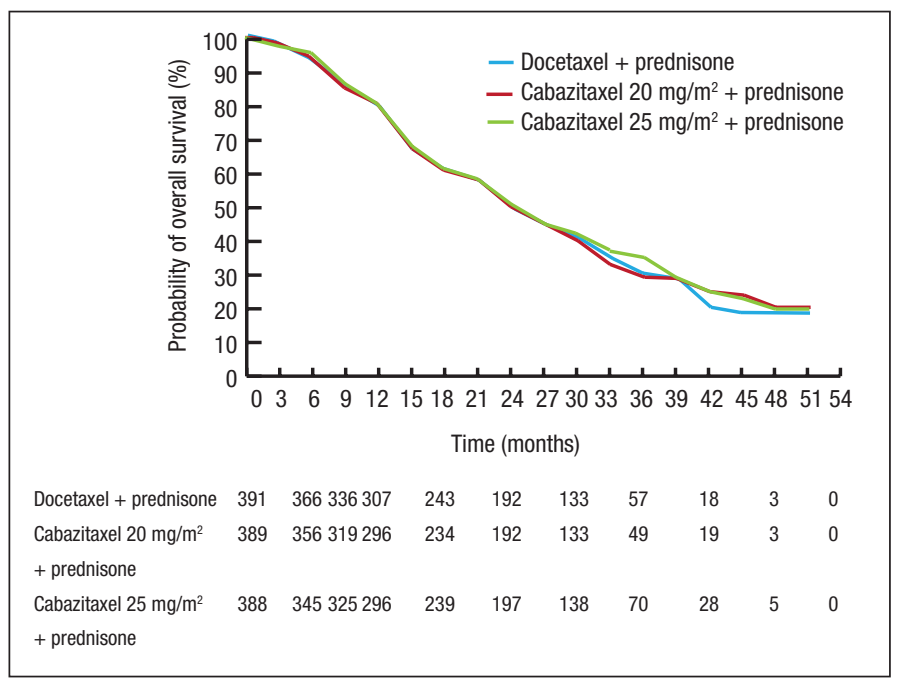

Fig.1. Overall survival in the FIRSTANA trial. ${ }^{10}$

maintain at least $50 \%$ of the OS benefit of the $25 \mathrm{mg} / \mathrm{m}^{2}$ dose relative to mitoxantrone observed in the TROPIC trial. The higher dose did not necessitate a lower delivered dose intensity (median 0.99 vs. 0.98) or decrease in the number of cycles administered ( 7 vs. 6) compared with the lower dose. However, more patients in the higher-dose group had a dose reduction by $1(21.5 \%$ vs. $10 \%)$. Very few patients in either arm had a second dose reduction. With a median OS of 13.4 vs. 14.5 months in the lower vs. higher dose groups (hazard ratio [HR] 1.024), the one-side $98.9 \%$ upperbound confidence interval $[\mathrm{Cl}]$ of 1.184 was within the noninferiority margin of 1.214. Of interest, in the subgroup of patients who had prior treatment with either abiraterone or enzalutamide, there was a trend toward improved survival with the higher dose of cabazitaxel; however, these differences did not reach statistical significance and the data are purely hypothesis-generating. There was no difference in PFS. The higher dose of cabazitaxel did result in a higher PSA response rate $(42.9 \%$ vs. $29.5 \% ; \mathrm{p}<0.0001)$ and a trend toward a higher RECIST response rate $(23.4 \%$ vs. $18.5 \%$; $\mathrm{p}=0.1924$ ), but was also associated with a higher rate of Grade 3-4 TEAEs than the lower dose, including higher rates of febrile neutropenia, hematuria, and diarrhea.

TAXYNERGY trial shows that early taxane switch may improve PSA response rate and that androgen receptor nuclear localization may indicate taxane sensitivity/resistance

In men with advanced prostate cancer, PSA declines with chemotherapy are associated with a survival advantage; ${ }^{12,13}$ however, approximately half of men with CRPC do not achieve PSA declines of $50 \%$ or greater with taxane-based chemotherapy. ${ }^{12}$ Because taxanes are not completely crossresistant, ${ }^{9,14}$ the randomized phase 2 TAXYNERGY trial explored whether an early taxane switch would benefit men 
who failed to achieve a sufficient PSA decline within four cycles of their original taxane therapy. ${ }^{15} \mathrm{~A}$ total of 63 men with chemotherapy-naïve $\mathrm{mCRPC}$ were randomly assigned in a 2:1 ratio to first-line docetaxel $(n=41)$ or cabazitaxel $(\mathrm{n}=22)$ and switched to the other taxane if their PSA did not decline by $30 \%$ or more by the fourth cycle. Otherwise, they remained on their original chemotherapy. Treatment was continued until progression, unacceptable toxicity, investigator decision, or study cut-off. Nearly one-third (29.3\%) of those who received first-line docetaxel and $13.6 \%$ of those who received first-line cabazitaxel did not achieve PSA declines of $30 \%$ or greater by Cycle 4 and were, therefore, switched to the alternative taxane. By study end, $55.6 \%$ of the overall population achieved a PSA response of $50 \%$ or greater; the predefined lower limit of $10 \%$ of the one-sided $\mathrm{Cl}$ was $47.5 \%$, which exceeded the historical control rate of $45.4 \%$ and was, therefore, positive.

The study also evaluated the association of biomarkers with taxane response/resistance by collecting circulating tumour cells (CTCs) at multiple time points — representing a real-time opportunity to investigate the mechanism of action (MOA) of taxanes. Analysis of CTCs for androgen receptor nuclear localization (ARNL) revealed that patients who experienced a $50 \%$ or greater decline in PSA had a mean decrease in ARNL of $6.5 \%$, compared with an increase of $6.1 \%$ in those who did not achieve a $50 \%$ PSA decline $(p=0.03)$. These results from TAXYNERGY suggest that further studies are warranted to identify the men with metastatic prostate cancer who might benefit from an early switch in taxane treatment. ARNL was identified as a potential marker of taxane sensitivity/resistance, which is consistent with the proposed MOA. Although interesting, these results should not yet change the way we routinely manage our patients with $\mathrm{mCRPC}$ who receive taxanes.

\section{Docetaxel monotherapy may generate a more rapid biochemical pro- gression after radical prostatectomy in patients with Gleason score 7 or lower}

Docetaxel has been shown to prolong survival in a number of advanced cancers, including breast, colorectal, and prostate cancer. In breast and colorectal cancer, a survival benefit has also been shown for docetaxel in the adjuvant setting. The multinational open-label phase 3 SPCG12 trial sought to determine whether a similar benefit would be seen for adjuvant docetaxel monotherapy in advanced prostate cancer. ${ }^{16}$ Following radical prostatectomy, 459 men with high-risk prostate cancer were randomly assigned to receive either six cycles of adjuvant docetaxel $75 \mathrm{mg} / \mathrm{m}^{2}$ every three weeks (in the absence of continuous steroids or ADT) for six weeks, or surveillance until their PSA levels reached $0.5 \mathrm{ng} /$ $\mathrm{mL}$. A higher than expected $14.2 \%$ rate of febrile neutropenia was observed, which may have been due to the lack of concomitant hormonal therapy and/or the recent surgery, creating a higher risk of infection. Biochemical progression, defined as a PSA level higher than $0.5 \mathrm{ng} / \mathrm{mL}$, was seen in $41.8 \%$ of the overall intent-to-treat population, with a trend toward greater progression in the docetaxel arm than in the surveillance arm ( $44.8 \%$ vs. $38.9 \%$; $p=0.078)$. Initially, the docetaxel arm saw a very low rate of progression; however, once docetaxel was stopped there was an increased rate of progression in the docetaxel arm compared with the surveillance arm, with the two curves crossing at 15 months. Beyond 24 months, the biochemical-free survival was consistently $10 \%$ lower in the docetaxel arm than in the surveillance arm (Fig. 2).

The strongest prognostic factors for biochemical progression were Gleason score of 8 or higher and lymph node metastases. Interestingly, the subgroup of patients with a Gleason score of 7 or lower fared significantly better with surveillance than with docetaxel monotherapy (HR 1.61) and there was a similar trend in the subgroup of patients with no lymph node metastases (HR 1.33). Overall, this study failed to prove the hypothesis that adjuvant docetaxel following radical prostatectomy provides a biochemical PFS advantage in men with high-risk prostate cancer. Instead, certain subgroups may actually fare better with active surveillance. The results of this study contrast those of the three previously reported post-radiation adjuvant studies that all showed an improvement in recurrence-free survival and immature OS estimates. ${ }^{17-19}$ Other than the local treatment modality used, the only major differences between this study and the other four is the lack of ADT used in the Scandinavian post-surgical study. More studies are needed to fully understand the role (if any) of chemotherapy in this population.

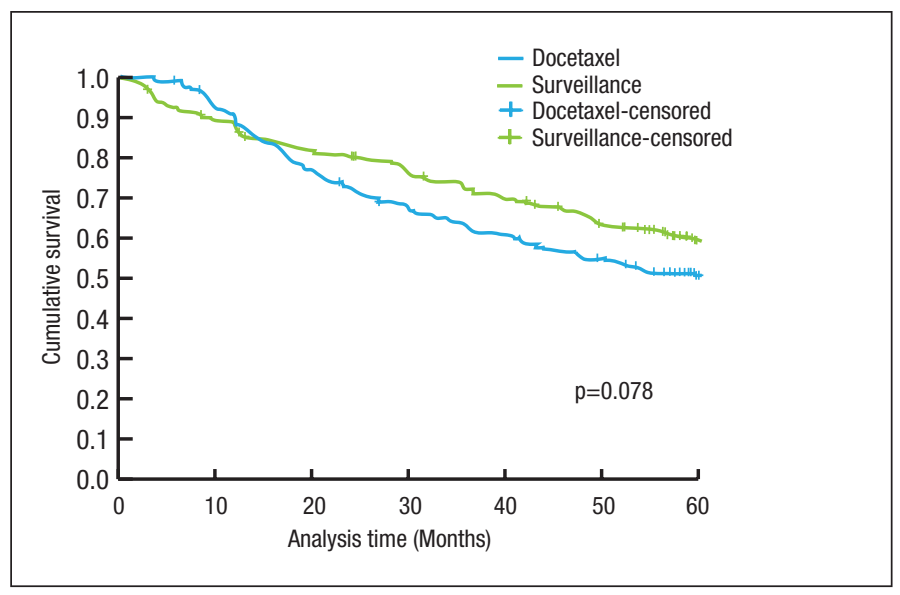

Fig. 2. Biochemical progression-free survival of patients randomly assigned to docetaxel monotherapy or surveillance following radical prostatectomy. ${ }^{16}$ 


\section{References}

1. Petrylak DP, Tangen CM, Hussain MH, et al. Docetaxel and estramustine compared with mitoxantrone and prednisone for advanced refractory prostate cancer. N Engl J Med 2004;351:1513-20. http://dx.doi.org/10.1056/NEJMoa041318

2. Tannock IF, de Wit R, Berry WR, et al; TAX 327 Investigators. Docetaxel plus prednisone or mitoxantrone plus prednisone for advanced prostate cancer. N Engl J Med 2004;351:1502-12. http://dx.doi. org/10.1056/NEJMoa040720

3. Patrick-Miller $U$. Is there a role for the assessment of health-related quality of life in the clinical evaluation of novel cytostatic agents?: Commentary re: P. M. LoRusso, Improvements in quality of life and diseaserelated symptoms in phase I trials of the selective oral epidermal growth factor receptor tyrosine kinase inhibitor ZD1839 in non-small cell lung cancer and other solid tumors. Clin Cancer Res 2003;9:2040-8. Clin Cancer Res 2003;9:1990-4.

4. Cella D, Peterman A, Hudgens $S$, et al. Measuring the side effects of taxane therapy in oncology: The functional assessment of cancer therapy-taxane (FACT-taxane). Cancer 2003;98:822-31. http://dx.doi. org/10.1002/cncr. 11578

5. Sweeney $\mathrm{CJ}$, Chen $\mathrm{YH}$, Carducci $\mathrm{M}$, et al. Chemohormonal therapy in metastatic hormone-sensitive prostate cancer. N Engl J Med 2015;373:737-46. http://dx.doi.org/10.1056/NEJMoa1503747

6. Patrick-Miller U, Chen Y-H, Carducci MA, et al. Quality of life (QOL) analysis from CHAARTED: Chemohormonal androgen ablation randomized trial in prostate cancer (E3805). J Clin Oncol 2016;34 (suppl). Abstr 5004.

7. Madan RA, Pal SK, Sartor 0 , et al. Overcoming chemotherapy resistance in prostate cancer. Clin Cancer Res 2011;17:3892-902. http://dx.doi.org/10.1158/1078-0432.CCR-10-2654

8. Cash H, Steiner U, Heidenreich A, et al. PRINCE: A phase 3 study comparing intermittent docetaxel therapy vs. continuous docetaxel therapy in patients with castration-resistant prostate cancer. J Clin Oncol 2016;34(suppl). Abstr 5005.

9. De Bono JS, Oudard S, Ozguroglu M, et al; TROPIC Investigators. Prednisone plus cabazitaxel or mitoxantrone for metastatic castration-resistant prostate cancer progressing after docetaxel treatment: A randomized, open-label trial. Lancet 2010;376:1147-54. http://dx.doi.org/10.1016/S01406736(10)61389-X

10. Sartor AO, Oudard S, Sengelov L, et al. Cabazitaxel vs docetaxel in chemotherapy-naive (CN) patients with metastatic castration-resistant prostate cancer (mCRPC): A three-arm, phase 3 study (FIRSTANA). J Clin Oncol 2016;34(suppl). Abstr 5006.
11. De Bono JS, Hardy-Bessard A-C, Kim C-S, et al. Phase 3 non-inferiority study of cabazitaxel (C) $20 \mathrm{mg} /$ m2 (C20) vs. $25 \mathrm{mg} / \mathrm{m} 2$ (C25) in patients (pts) with metastatic castration-resistant prostate cancer (mCRPC) previously treated with docetaxel (D). J Clin Oncol 2016;34(suppl). Abstr 5008.

12. Armstrong AJ, Garrett-Mayer E, Ou Yang YC, et al. Prostate-specific antigen and pain surrogacy analysis in metastatic hormone-refractory prostate cancer. J Clin Oncol 2007;25:3965-70. http://dx.doi. org/10.1200/JC0.2007.11.4769

13. Petrylak DP, Ankerst DP, Jiang CS, et al. Evaluation of prostate-specific antigen declines for surrogacy in patients treated on SWOG 99-16. J Natl Cancer Inst 2006;98:516-21. http://dx.doi.org/10.1093/ inci/dij129

14. van Soest RJ, de Morrée ES, Kweldam CF, et al. Targeting the androgen receptor confers in vivo crossresistance between enzalutamide and docetaxel, but not cabazitaxel, in castration-resistant prostate cancer. Eur Urol 2015;67:981-5. http://dx.doi.org/10.1016/i.eururo.2014.11.033

15. Tagawa ST, Antonarakis ES, Saad F, et al. TAXYNERGY: Randomized trial of early switch from first-line docetaxel (D) to cabazitaxel (C) or vice versa with circulating tumour cell (CTC) biomarkers in patients (pts) with metastatic castration-resistant prostate cancer (mCRPC). J Clin Oncol 2016;34(suppl). Abstr 5007.

16. Ahlgren $\mathrm{G}$, Flodgren $\mathrm{P}$, Tammela TL, et al. A randomized, phase 3 trial between adjuvant docetaxel and surveillance after radical prostatectomy for high-risk prostate cancer: Results of SPCG12. J Clin Oncol 2016;34(suppl). Abstr 5001.

17. Sandler HM, Hu C, Rosenthal SA, et al. A phase 3 protocol of androgen suppression (AS) and 3DCRT/ IMRT vs. AS and 3DCRT/IMRT followed by chemotherapy (CT) with docetaxel and prednisone for localized, high-risk prostate cancer (RTOG 0521). J Clin Oncol 2015;33(suppl). Abstr LBA5002.

18. Fizazi K, Faivre L, Lesaunier $F$, et al. Androgen-deprivation therapy plus docetaxel and estramustine vs. androgen-deprivation therapy alone for high-risk localised prostate cancer (GETUG 12): A phase 3, randomized, controlled trial. Lancet Oncol 2015;16:787-94. http://dx.doi.org/10.1016/S14702045(15)00011-X

19. James ND, Sydes MR, Clarke NW, et al; STAMPEDE investigators. Addition of docetaxel, zoledronic acid, or both to first-line long-term hormone therapy in prostate cancer (STAMPEDE): Survival results from an adaptive, multiarm, multistage, platform-randomized, controlled trial. Lancet 2016;387:1163-77. http://dx.doi.org/10.1016/S0140-6736(15)01037-5 